\title{
Opinions on Paleolithic physiology living in painogenic environments: changing the perspective through which we view chronic pain
}

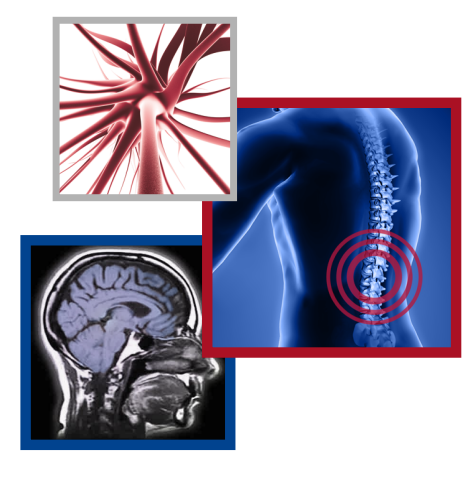

Mark I Johnson*,1

${ }^{1}$ Centre for Pain Research, School of Clinical and Applied Sciences, Leeds Beckett University, Leeds, West Yorkshire, LS1 3HE, UK

*Author for correspondence: M.Johnson@Leedsbeckett.ac.uk

"Comparing the lifestyles of Paleolithic and Anthropocene humans from an evolutionary mismatch perspective may offer novel insights into the origin and management of chronic pain in modern times"

First draft submitted: 20 December 2018; Accepted for publication: 15 February 2019; Published online: 29 May 2019

Keywords: chronic pain • modern urban living • Paleolithic

\section{Context}

Previously, in Pain Management my colleagues and I revealed a prominence of pain education, but a lack of health promotion discourse in mainstream pain journals [1]. We argued that knowledge about pain was one of many complex factors affecting the choices people make about their health and that pain education was "...very much downstream by managing the situation once pain has become a problem." We suggested that upstream activities focusing on prevention would have a greater impact on society's burden of chronic pain. We reasoned that a 'social model' of health promotion that reduces social inequality (e.g., poverty, poor housing and lack of employment) would create social conditions promoting health and wellbeing. We concluded, "...it is not fair simply to tell people of the 'right' actions to take to produce healthy outcomes but it is also incumbent upon policy makers to provide the means to put those decisions into practice." In this article, I would like to extend this notion by speculating that discrepancies between physiology inherited from our evolutionary heritage (Paleolithic physiology) and lifestyles played out in present day urban environments may be perpetuating chronic pain. I will argue that present-day lifestyles and urban environments are painogenic in nature and that a focus on evolutionary perspectives may offer solutions to reduce chronic pain in the future. At present, evidence in this field is limited and therefore a speculative outlook is provided.

\section{Setting out the problem}

The prevalence of chronic pain is high. In 2012, our team estimated the weighted mean + standard deviation prevalence of chronic pain in adults worldwide to be $30.3 \%+11.7 \%$ (19 studies, 65 surveys, $\overline{34}$ countries, 182,019 respondents) [2]. In 2016, Fayaz et al. [3] estimated the prevalence of chronic pain in UK adults to be between 38.4 and 48.6\% (95\% CI, seven studies), although the prevalence of moderate to severely disabling chronic pain was between 10.4 and $14.3 \%$ (95\% CI, four studies). Mean prevalence increased with age from 14.3\% in 18-25 years old, to $62 \%$ in over 75 year olds. This trend is likely to get worse with an aging population.

The International Classification of Diseases version-11 (ICD-11) of the WHO categorizes chronic pain (MG30) according to its primary cause as chronic cancer-related pain, chronic postsurgical and post-traumatic pain, chronic secondary musculoskeletal pain, chronic secondary visceral pain, chronic neuropathic pain and chronic secondary headache and orofacial pain [4]. In addition, ICD-11 includes a new phenomenological coding to capture an increasing number of conditions that defy traditional classification based on etiology or pathophysiology. This new coding is chronic primary pain and includes conditions such as chronic widespread pain, fibromyalgia, irritable bowel syndrome and chronic nonspecific low back pain. 
Management of chronic pain remains a challenge. Patients with similar physical insults, diseases and treatment approaches may present with quite varied pain experience, response to treatment and long-term outcome. A biopsychosocial stepped model of care is desirable, although there continues to be an over-reliance on biomedical models of care for chronic long-term illness and this may have harmful consequences for patients through overdiagnosis and overprescribing [5]. For example, in USA, open-ended prescribing of opioids for chronic pain has contributed to the rise in opioid abuse and overdose deaths associated with the 'opioid epidemic' [6]. Paradoxically, in parts of Asia, Africa and the Middle East restricted access to opioids hinders appropriate pain management.

The importance of social factors in pain experience is undisputable, and recently there have been calls to re-define the International Association for the Study of Pain's (IASP) definition of pain to include social factors as follows: "... a distressing experience associated with actual or potential tissue damage with sensory, emotional, cognitive, and social components [7]." In clinical practice, healthcare professionals offer advice to patients about the impact of social components on pain. Professional body guidelines recommend that healthcare professionals counsel patients experiencing chronic pain to adopt the 'right' lifestyle actions to produce healthy outcomes including physical activity and balanced diets [8]. Evidence suggests that many patients with long-term chronic illness fail to adhere to advice about healthy lifestyles, especially in relation to physical activity and healthy diet [9]. One reason for failure to achieve long-term lifestyle change may be that the environment in which life is lived mitigates healthy living. Modern lifestyles in urban environments have been associated with obesity (i.e., obesogenic environments). Could modern urban environments also be 'painogenic' in nature?

\section{Painogenicity of the urban environment}

The concept of obesogenic (obesity-promoting) environments arose in the late 1990s to draw attention to the association between the built and food environment in order to tackle obesity through public health and the design of urban environments (i.e., whole systems approaches) [10]. Swinburn et al. defined the obesogenicity of an environment as the sum of influences that the surroundings, opportunities or conditions of life have on promoting obesity in individuals or populations [11]. There are parallels between obesity and chronic pain. Both conditions are influenced by biopsychosocial factors including physical activity and diet, and are managed using pharmacological, educational and behavioral interventions with limited overall success; both conditions have high economic and social costs and contribute to health inequalities by disproportionately affecting poorer people in society [12].

Discourse on painogenicity of the urban environment is scarce. A definition of a painogenic environment could be the sum of influences that the surroundings, opportunities or conditions of life have on promoting persistent pain in individuals or populations, in line with Swinburn et al.'s definition for obesogenic environments [11]. Living within a painogenic environment increases the likelihood of exposure to factors that have the potential to increase the frequency and/or severity and/or persistence of pain. Examples include sedentary behavior, diets of excess fat, sucrose, salt and additives from overprocessed foods, use of recreational drugs (e.g., alcohol, tobacco), prescription medication, low socioeconomic status, income inequalities, poor working conditions, unemployment, unstable home life, low levels of education and living in deprived environments [13]. Whether a person ultimately experiences pain, suffering and disability depends on a multitude of other factors including their ability to cope.

There is growing interest in the role of lifestyle factors on chronic pain, but rarely from an evolutionary perspective. Consideration of the evolutionary heritage of humans has provided insights into the biological role of sensitization and hyperalgesia in chronic pain $[14,15]$. Evolutionary perspectives have also provided insights into the influence of lifestyle and environment on noncommunicable diseases by comparing Paleolithic physiology with Anthropocene environments. To my knowledge, there has been no comparison between Paleolithic physiology and Anthropocene environment in the context of chronic pain.

\section{Paleolithic physiology living in Anthropocene environments}

The genetic material in modern humans encodes physiology adapted for hominin ancestors that existed in the Paleolithic era, circa 7-4 million years ago. Paleolithic humans lived in environments with air free from toxic emissions and particulates from burning fossil fuels, with a daily diet of fresh vegetables, fruit and raw meat and a routine of walking, climbing, lifting, carrying and bending (i.e., hunter-gatherer lifestyles). The Agricultural Revolution (12,000-15,000 years ago) catalyzed a dominance of human activity on the environment and the Earth's ecosystems including climate (i.e., the Anthropocene era). Nowadays, many humans live in urbanized built environments (i.e., cities, towns, conurbations or suburbs) with limited greenspace and air contaminated by 
human-made pollutants. Many urban dwellers have diminishing levels of physical activity due to sedentary jobs and reliance on motorized vehicles, and consume overprocessed foods with excess sucrose, salt, fat and additives.

The evolutionary mismatch hypothesis explains how the shift to modern urban living has been more rapid than the time needed for physiological mechanisms to adapt to the change. It has been suggested that evolutionary advantageous traits provided by Paleolithic physiology may contribute to noncommunicable 'lifestyle diseases' ( $c f$. sick city syndrome, modern urban living syndrome) [16]. Lindeberg and Lundh [17] investigated 23,000 inhabitants on Kitava Island living hunter-gatherer lifestyles on a diet of fish, fruit, root and other vegetables, and found no incidences of death from stroke, ischemic heart disease, diabetes or obesity. In contrast, deaths from noncommunicable diseases such as cardiovascular diseases (heart attacks and stroke), chronic respiratory diseases (chronic obstructed pulmonary disease and asthma), diabetes and cancer are common in dwellers of modern urbanized settings [18]. The United Nations predict that urban populations will exceed 6.4 billion by 2050 (i.e., circa $65 \%$ of the global population), with deaths associated with chronic noncommunicable diseases projected to increase accordingly [19].

Chronic pain often accompanies noncommunicable diseases causing suffering, disability and a significant impact on quality of life. For example, the Global Burden of Disease Study 2013, found that chronic low back pain had the highest number of years lived with disability. Risk factors for noncommunicable diseases includes lifestyle behavior such as sedentary activity, unhealthy diet, anxiety and depression, smoking, lack of sunshine, disrupted sleep, unemployment, living in adverse socioeconomic circumstances and previous history of abuse or violence [20]. These factors are also associated with chronic pain, although the picture is complex with causal processes acting in both directions $[13,21]$. Comparing the lifestyles of Paleolithic and Anthropocene humans from an evolutionary mismatch perspective may offer novel insights into the origin and management of chronic pain in modern times. It is important to emphasize that in doing so one should not assume that Paleolithic humans had a lower incidence or severity of chronic pain.

\section{Physical activity: Paleolithic versus Anthropocene}

Humans are genetically adapted for a daily routine of light-to-moderate activity, essential for survival as huntergatherers in rural environments. The physical nature of hunter-gatherer lives depends on manual chores associated with regular walking, bending, climbing, lifting and carrying loads such as wood, water, food and children. The typical daily distance covered by hunter-gatherers is between 3 and 10 miles with an average energy expenditure of daily activities between 3000 and $5000 \mathrm{~kJ}$, five-times greater than modern sedentary adults [22]. Modern sedentary lifestyle involves excessive amounts of time being physically inactive resulting in an evolutionary mismatch manifesting as 'disuse syndromes' ('walking deficiency syndrome', 'hyper-sitting syndrome') [23]. Sedentary lifestyles are associated with increased risk of heart disease, obesity and Type 2 diabetes, and chronic pain including nonspecific chronic musculoskeletal pain [23,24]. For nonspecific chronic musculoskeletal pain, clinical guidelines recommend lifestyle adjustment with physical activity at its core (e.g., for nonspecific chronic low back pain [25]). This may be challenging to achieve if the environment is not conducive to outdoor activity due to air and noise pollution and limited access to greenspace. Taking up physical activities may introduce additional problems such as chronic overuse injuries such as strains and tendonitis from, for example, jogging on concrete or treadmills in restrictive shoes [16].

\section{Diet: Paleolithic versus Anthropocene}

The diet of early humans comprised a mixed diet of fruit, tough vegetation and raw meat obtained from scavenging. The advent of cooking (circa 1 million years ago) improved the digestibility of meat and plants, and the advent of agriculture and animal husbandry (circa 12,000 years ago) and this enabled calorie intake to exceed growth and energy requirements of humans. There have been many positive consequences associated with this shift in diet, although one negative consequence was a decline in the diversity of nutritional intake. In recent times, this has been further compounded as urban dwellers consume fewer vegetables, fruits, antioxidants and omega-3 fatty acids and increasing amounts of fat, sucrose, salt and additives in overprocessed foods. This evolutionary mismatch has been associated with diet-induced proinflammatory states: "we literally eat ourselves into an inflamed and painful state and then seek out passive care from doctors to intervene on our behalf" [26].

Research on the influence of nutrition and diet on chronic pain has been gathering pace with increasing emphasis on the importance of diet therapy in the management of chronic pain [27]. Evidence suggests that chronic pain can be alleviated by increasing consumption of antioxidants and omega- 3 fatty acids, and reducing consumption of exocitoxic substances found in additives (e.g., monosodium glutamate, hydrolyzed protein, protein 
isolates/concentrates, yeast extract, aspartame) and foodstuffs (e.g., bran, nuts, soybean and aged cheeses) [28]. Adopting a healthy diet can be very challenging in modern urban environments where overprocessed foodstuffs are inexpensive, readily available and seen as the norm.

\section{Human microbiota: Paleolithic versus Anthropocene}

Evolution of our genetic heritage produced Paleolithic humans adapted to exist in environments with direct exposure to soil, plants and animals. An array of microorganisms thrived on the skin and within the GI tract of Paleolithic humans (i.e., the human microbiota). There is a symbiotic relationship between the human microbiota and the immune system to enable appropriate and timely protective responses to pathogens and tolerance to innocuous antigens. The human GI-microbiota-brain axis regulates a variety of physiological processes including immune priming and protection against invading pathogens [29]. Disruption to the composition of the GI microbiota weakens immune responses triggering allergic responses to harmless allergens, although mechanisms are complex and poorly understood.

The majority of modern urban dwellers spend excessive amounts of time living indoors with artificially conditioned air, eating and drinking processed foodstuffs, and using pharmaceuticals to eradicate infections and parasites. Humans acquire their microbiota after birth and children with minimal exposure to microorganisms develop immune systems that are unable to recognize or differentiate pathogens that confer benefit or harm [30]. Thus, diminished exposure to microorganisms contributes to low microbial diversity in the human microbiota creating an evolutionary mismatch. A compromised GI microbiota is associated with a variety of noncommunicable diseases including inflammatory bowel disease, celiac disease, metabolic syndrome obesity, asthma, Type 2 diabetes and various brain disorders, many of which are associated with chronic pain [29]. Recently, attention has focused on the role of the GI microbiota in the regulation of visceral pain and the therapeutic potential of dietary interventions such as probiotics and prebiotics to alleviate visceral hypersensitivity [31].

\section{Moving chronic pain upstream}

Physical activity and diet modification have become central in the support of analgesic therapy for many chronic pain conditions. Clinical guidelines command healthcare professionals to counsel pain patients to adopt the 'right' actions to produce healthy outcomes, but patient adherence is poor and relapse common. A meta-ethnography found that patients with chronic nonmalignant musculoskeletal pain experience a constant adversarial struggle with healthcare systems [32]. The disconnect between healthy lifestyle advice and the environment in which lifestyle is lived may add to that adversary struggle. Our Paleolithic ancestors were adapted to exist in calorie-limited environments by minimizing energy expenditure and maximizing calorific consumption through cravings for fat, sugar and salt and modern urban environments promote sedentary behaviors and consumption of overprocessed foods. Thus, it is hardly surprising that chronic pain patients struggle to follow healthy lifestyle advice given by healthcare professionals. In fact, the socioeconomic pressures of busy modern lifestyles may mean that many patients no longer identify with 'being active' or 'healthy eating' and have given up the struggle to sustain regular exercise and healthy diets.

Currently, healthcare policy advocates a biopsychosocial approach to manage chronic pain, but often service delivery defaults to medical models of care using prescriptions of 'drugs', 'exercise' or 'diet'. Reasons for this are complex. There have been calls to reframe the model of care by shifting emphasis further upstream, although introducing population-level solutions is challenging and continuation of a medical model of care may suit some stakeholders [33]. Healthcare professionals may believe that population-level solutions are beyond their remit. It is incumbent on healthcare policy makers adopt upstream strategies that focus on prevention to reduce the burden of chronic pain (and noncommunicable diseases) on society. In February 2014, the Evolutionary Determinants of Health program was launched to provide a framework to debate the design of evolutionary-concordant healthy cities and social regimes for urban societies of the future [34]. Evolutionary-concordant urban environments encourage individuals to be physically activity through the provision of greenspace, clean air, foodstuffs that encourage balanced diets and better social conditions that reduce social injustices associated with poverty, poor housing and lack of employment.

\section{Conclusion}

Exploring the management and prevention of chronic pain through an evolutionary lens is rare. 
In this article, I have taken a speculative approach to explore the impact of the mismatch between Paleolithic physiology and modern urban environments on chronic pain. This evolutionary mismatch perspective offers opportunities to investigate painogenicity of built and food environments and the challenges faced in current practice, such as why patients experience difficulties adhering to clinical recommendations related to lifestyle adjustment. The evolutionary mismatch perspective also offers upstream strategies to reduce the burden of chronic pain on society through the design of evolutionary-concordant urban environments. I hope that this article catalyzes further research and scholarship in this field.

Acknowledgments

I wish to express gratitude to the anonymous referees and editors for their constructive advice and to G Jones and P Wittkopf for commenting on the revised manuscript.

Financial \& competing interests disclosure

MI Johnson's institution has received payments from GlaxoSmithKline for consultancy work undertaken by him. The author has no other relevant affiliations or financial involvement with any organization or entity with a financial interest in or financial conflict with the subject matter or materials discussed in the manuscript apart from those disclosed.

No writing assistance was utilized in the production of this manuscript.

Accessing research materials

Underlying research materials related to our paper (for example data, samples or models) can be accessed by contacting MI Johnson.

\section{References}

1. Johnson MI, Briggs M, Dixey R. Should health promotion be on the pain agenda? Pain. Manag. 4(6), 385-388 (2014).

2. Elzahaf RA, Tashani OA, Unsworth BA, Johnson MI. The prevalence of chronic pain with an analysis of countries with a Human Development Index less than 0.9: a systematic review without meta-analysis. Curr. Med. Res. Opin 28(7), 1221-1229 (2012).

3. Fayaz A, Croft P, Langford RM, Donaldson LJ, Jones GT. Prevalence of chronic pain in the UK: a systematic review and meta-analysis of population studies. BMJ Open 6(6), e010364 (2016).

4. WHO. The International Classification of Diseases version-11 of the World Health Organization. https://icd.who.int

5. Le Fanu J. Mass medicalisation is an iatrogenic catastrophe. BMJ 361, k2794 (2018).

6. Shipton EA, Shipton EE, Shipton AJ. A review of the opioid epidemic: what do we do about it? Pain Ther. 7(1), 23-36 (2018).

7. Williams AC, Craig KD. Updating the definition of pain. Pain 157(11), 2420-2423 (2016).

8. National Institute for Health and Care Excellence (NICE). Physical activity: brief advice for adults in primary care. Public Health Guideline [PH44] (2013). www.nice.org.uk/guidance/ph44

9. King DE, Mainous AG, 3rd, Carnemolla M, Everett CJ. Adherence to healthy lifestyle habits in US adults, 1988-2006. Am. J. Med. 122(6), 528-534 (2009).

10. Townshend T, Lake A. Obesogenic environments: current evidence of the built and food environments. Perspect. Public Health 137(1), 38-44 (2017).

11. Swinburn B, Egger G, Raza F. Dissecting obesogenic environments: the development and application of a framework for identifying and prioritizing environmental interventions for obesity. Prev. Med. 29(6), 563-570 (1999).

12. Riskowski JL. Associations of socioeconomic position and pain prevalence in the United States: findings from the National Health and Nutrition Examination Survey. Pain Med. 15(9), 1508-1521 (2014).

13. Van Hecke O, Torrance N, Smith BH. Chronic pain epidemiology - where do lifestyle factors fit in? Br. J. Pain 7(4), 209-217 (2013).

14. Price TJ, Dussor G. Evolution: the advantage of 'maladaptive' pain plasticity. Curr. Biol. 24(10), R384-R386 (2014).

15. Williams AC. What can evolutionary theory tell us about chronic pain? Pain 157(4), 788-790 (2016).

16. Lieberman DE. The Story of the Human Body: Evolution, Health and Disease. Penguin Books, London, UK (2014).

17. Lindeberg S, Lundh B. Apparent absence of stroke and ischaemic heart disease in a traditional Melanesian island: a clinical study in Kitava. J. Intern. Med. 233(3), 269-275 (1993).

18. WHO. The top 10 causes of death. www.who.int/en/news-room/fact-sheets/detail/the-top-10-causes-of-death

19. United Nations. 2018 Revision of World Urbanization Prospects. www.un.org/development/desa/publications/2018-revision-of-world-urbanization-prospects.html

20. Smuck M, Kao MC, Brar N, Martinez-Ith A, Choi J, Tomkins-Lane CC. Does physical activity influence the relationship between low back pain and obesity? Spine J. 14(2), 209-216 (2014). 
21. Dean E, Soderlund A. What is the role of lifestyle behaviour change associated with non-communicable disease risk in managing musculoskeletal health conditions with special reference to chronic pain? BMC Musculoskeletal Disord. 16, 87 (2015).

22. Cordain L, Gotshall RW, Eaton SB, Eaton SB. Physical activity, energy expenditure and fitness: an evolutionary perspective. Int. J. Sports Med. 19(5), 328-335 (1998).

23. Van Wilgen CP, Dijkstra PU, Versteegen GJ, Fleuren MJ, Stewart R, Van Wijhe M. Chronic pain and severe disuse syndrome: long-term outcome of an inpatient multidisciplinary cognitive behavioural programme. J. Rehabil. Med. 41(3), 122-128 (2009).

24. Chen SM, Liu MF, Cook J, Bass S, Lo SK. Sedentary lifestyle as a risk factor for low back pain: a systematic review. Int. Arch. Occup. Environ. Health. 82(7), 797-806 (2009).

25. National Institute for Health and Care Excellence (NICE). Low back pain and sciatica in over 16s: assessment and management. Clinical Guideline [NG59]. 1-18 (2016). www.nice.org.uk/guidance/ng59

26. Seaman DR. The diet-induced proinflammatory state: a cause of chronic pain and other degenerative diseases? J. Manipulative Physiol. Ther. 25(3), 168-179 (2002).

27. Rondanelli M, Faliva MA, Miccono A et al. Food pyramid for subjects with chronic pain: foods and dietary constituents as anti-inflammatory and antioxidant agents. Nutr. Res. Rev. 31(1), 131-151 (2018).

28. Brain K, Burrows TL, Rollo ME et al. A systematic review and meta-analysis of nutrition interventions for chronic noncancer pain. $J$. Human Nut. Dietetics 32(2), 198-225 (2019).

29. Young VB. The role of the microbiome in human health and disease: an introduction for clinicians. BMJ 356, j831 (2017).

30. Rook GA. Regulation of the immune system by biodiversity from the natural environment: an ecosystem service essential to health. Proc. Natl Acad. Sci. USA 110(46), 18360-18367 (2013).

31. Rea K, O’Mahony SM, Dinan TG, Cryan JF. The role of the gastrointestinal microbiota in visceral pain. Handb. Exp. Pharmacol. 239, 269-287 (2017).

32. Toye F, Seers K, Allcock N et al. A meta-ethnography of patients' experience of chronic non-malignant musculoskeletal pain. Health Serv. Deliv. Res. 1(12), (2013).

33. Lewis J, O'Sullivan P. Is it time to reframe how we care for people with non-traumatic musculoskeletal pain? Br. J. Sports Med. 52(24), 1543-1544 (2018).

34. Milne G. The evolutionary determinants of health programme: urban living in the 21 st Century from a human evolutionary perspective. Archaeol. Int. 18, 84-96 (2015). 